\title{
Riesz transforms and multipliers for the Grushin operator
}

\author{
K. Jotsaroop, P. K. Sanjay and S. Thangavelu
}

\begin{abstract}
We show that Riesz transforms associated to the Grushin operator $G=-\Delta-|x|^{2} \partial_{t}^{2}$ are bounded on $L^{p}\left(\mathbb{R}^{n+1}\right)$. We also establish an analogue of Hörmander-Mihlin multiplier theorem and study Bochner-Riesz means associated to the Grushin operator. The main tools used are Littlewood-Paley theory and an operator valued Fourier multiplier theorem due to L. Weis.
\end{abstract}

\section{Introduction}

The aim of this paper is to study Riesz transforms and multipliers associated to the Grushin operator $G=-\Delta-|x|^{2} \partial_{t}^{2}$ on $\mathbb{R}^{n} \times \mathbb{R}$. The spectral decomposition of this non-negative operator is explicitly known:

$$
G f(x, t)=\frac{1}{2 \pi} \int_{-\infty}^{\infty} e^{-i \lambda t}\left(\sum_{k=0}^{\infty}(2 k+n)|\lambda| P_{k}(\lambda) f^{\lambda}(x)\right) d \lambda
$$

where $P_{k}(\lambda)$ are the spectral projections of the scaled Hermite operator $H(\lambda)=-\Delta+\lambda^{2}|x|^{2}$ so that

$$
H(\lambda)=\sum_{k=0}^{\infty}(2 k+n)|\lambda| P_{k}(\lambda)
$$

and

$$
f^{\lambda}(x)=\int_{-\infty}^{\infty} f(x, t) e^{i \lambda t} d t
$$

1991 Mathematics Subject Classification. 42C, 42C10, 43A90. 
Thus, given a bounded function $m$ on $\mathbb{R}$ we can define the multiplier transformation $m(G)$ by setting

$$
m(G) f(x, t)=\frac{1}{2 \pi} \int_{-\infty}^{\infty} e^{-i \lambda t}\left(\sum_{k=0}^{\infty} m((2 k+n)|\lambda|) P_{k}(\lambda) f^{\lambda}(x)\right) d \lambda .
$$

It is obvious that $m(G)$ is bounded on $L^{2}\left(\mathbb{R}^{n+1}\right)$ but for the boundedness of $m(G)$ on other $L^{p}$ spaces we need to assume more conditions on $m$.

Recall that $H(\lambda)$ can be written as

$$
H(\lambda)=\frac{1}{2} \sum_{j=1}^{n}\left(A_{j}(\lambda) A_{j}(\lambda)^{*}+A_{j}(\lambda)^{*} A_{j}(\lambda)\right)
$$

where

$$
A_{j}(\lambda)=-\frac{\partial}{\partial x_{j}}+\lambda x_{j}, A_{j}(\lambda)^{*}=\frac{\partial}{\partial x_{j}}+\lambda x_{j}
$$

are the creation and annihilation operators (for $\lambda>0$ ). The operators

$$
R_{j}(\lambda)=A_{j}(\lambda) H(\lambda)^{-\frac{1}{2}}, R_{j}^{*}(\lambda)=A_{j}(\lambda)^{*} H(\lambda)^{-\frac{1}{2}}
$$

are Riesz transforms associated to the Hermite operator. It is therefore natural to consider the operators

$$
R_{j} f(x, t)=\frac{1}{2 \pi} \int_{-\infty}^{\infty} e^{-i \lambda t} R_{j}(\lambda) f^{\lambda}(x) d \lambda
$$

and $R_{j}^{*}$ similarly defined in terms of $R_{j}^{*}(\lambda)$ and call them the Riesz transforms for the Grushin operators. Again these Riesz transforms are trivially bounded on $L^{2}$ but their $L^{p}$ boundedness is far from trivial. We prove:

Theorem 1.1. For $1<p<\infty$ the Riesz transforms $R_{j}, R_{j}^{*}, j=$ $1,2, \ldots, n$ are all bounded on $L^{p}\left(\mathbb{R}^{n+1}\right)$.

We also consider higher order Riesz transforms which are defined as follows. Let $\mathcal{H}_{p, q}$ stand for the space of bigraded solid harmonics of bidegree $(p, q), p, q \in \mathbb{N}$. Let $G_{\lambda}(P)$ stand for the Weyl correspondence, see Section 2.2 for the definition. Then it is known that $G_{\lambda}(P) H(\lambda)^{-(p+q) / 2}$ are bounded operators on $L^{p}\left(\mathbb{R}^{n}\right), 1<p<\infty[\mathbf{6}]$. We define higher order Riesz transforms for the Grushin operator by setting

$$
R_{P} f(x, t)=\frac{1}{2 \pi} \int_{-\infty}^{\infty} e^{-i \lambda t} G_{\lambda}(P) H(\lambda)^{-(p+q) / 2} f^{\lambda}(x) d \lambda
$$

We prove: 
Theorem 1.2. For any $P \in \mathcal{H}_{p, q}$ the Riesz transforms $R_{P}$ are bounded on $L^{p}\left(\mathbb{R}^{n+1}\right), 1<p<\infty$.

Riesz transforms and higher order Riesz transforms associated to the sublaplacian on the Heisenberg group $\mathbb{H}^{n}$ has been studied by several authors, see e.g. [1],[2] and [7]. Riesz transforms on $\mathbb{H}^{n}$ and more generally on nilpotent Lie groups turn out to be singular integral operators. As we do not have a group structure behind the Grushin operator it is not possible to use their techniques. We use a different method described below. Concerning general multiplier transforms $m(G)$ we prove the following result.

Theorem 1.3. Let $N \geq \frac{n}{2}+1$ be an integer and let $m \in C^{N}\left(\mathbb{R}^{*}\right)$ satisfy the estimates $\left|m^{(k)}(\lambda)\right| \leq C_{k}|\lambda|^{-k}$ for $|\lambda|$ large for all $k=0,1,2, . ., N$. Then $m(G)$ is bounded on $L^{p}\left(\mathbb{R}^{n+1}\right)$ for all $1<p<\infty$.

In his thesis [5] R. Meyer has studied the wave equation associated to the Grushin operator in one dimension. There he mentions about the possibility of proving a multiplier theorem (stated as a conjecture) for the Grushin operator. The above theorem gives such a result though the proof is completely different from what he had in mind. We also have results for the wave equation which will be presented in a forthcoming paper.

We can also treat Bochner-Riesz means associated to the Grushin operator. For $R>0$ Bochner-Riesz means $B_{R}^{\delta} f$ of order $\delta>0$ are defined by

$$
B_{R}^{\delta} f(x, t)=\frac{1}{2 \pi} \int_{-\infty}^{\infty} e^{-i \lambda t}\left(1-\frac{H(\lambda)}{R}\right)_{+}^{\delta} f^{\lambda}(x) d \lambda .
$$

Here $\left(1-\frac{H(\lambda)}{R}\right)_{+}^{\delta} f^{\lambda}$ are the Bochner-Riesz means associated to $H(\lambda)$. For $\lambda$ fixed these means have been studied by various authors, see [9]. Concerning $B_{R}^{\delta} f$ for the Grushin operator we prove the following result.

Theorem 1.4. For $\delta>(n+1) / 2+1 / 6$, the Bochner-Riesz means $B_{R}^{\delta}$ are uniformly bounded on $L^{p}\left(\mathbb{R}^{n+1}\right), 1<p<\infty$.

It may not be possible to improve the above result when $n=1$ as the critical index for the Bochner-Riesz summability of one dimensional Hermite expansions is $1 / 6$, see [9]. However, the critical index for Hermite expansions on $\mathbb{R}^{n}, n \geq 2$ is $(n-1) / 2$ and hence it should be possible to improve the above result. We conjecture that the above result is true for $\delta>(n+1) / 2$ for $n \geq 2$. 

If

We now briefly describe the methods used to prove these theorems.

$$
m(H(\lambda))=\sum_{k=0}^{\infty}(2 k+n)|\lambda| P_{k}(\lambda)
$$

is the multiplier transform for the Hermite operator then it follows that

$$
(m(G) f)^{\lambda}(x)=m(H(\lambda)) f^{\lambda}(x) .
$$

Therefore, if we identity $L^{p}\left(\mathbb{R}^{n+1}\right)$ with $L^{p}(\mathbb{R}, X)$ where $X=L^{p}\left(\mathbb{R}^{n}\right)$ then we can view $m(G)$ as an operator valued Fourier multiplier (for the Fourier transform on $\mathbb{R})$ acting on $L^{p}(\mathbb{R}, X)$. Sufficient conditions on $m$ are known so that such multipliers are bounded on $L^{p}(\mathbb{R}, X)$. Indeed, we make use of the following theorem of L. Weis [12].

Theorem 1.5. Let $X$ and $Y$ be UMD spaces. Let $m: \mathbb{R}^{*} \rightarrow B(X, Y)$ be a differentiable function such that the families $\left\{m(\lambda): \lambda \in \mathbb{R}^{*}\right\}$ and $\left\{\lambda \frac{d}{d \lambda} m(\lambda): \lambda \in \mathbb{R}^{*}\right\}$ are $R$-bounded. Then $m$ defines a Fourier multiplier which is bounded from $L^{p}(\mathbb{R}, X)$ into $L^{p}(\mathbb{R}, Y)$ for all $1<$ $p<\infty$.

We need this theorem only for $X=Y=L^{p}\left(\mathbb{R}^{n}\right)$ and in this case the R-boundedness is equivalent to a vector-valued inequality for $m(\lambda)$ and $\lambda \frac{d}{d \lambda} m(\lambda)$. Indeed, the R-boundedness of a family of operators $T(\lambda)$ is equivalent to the inequality

$$
\left\|\left(\sum_{j=1}^{\infty}\left|T\left(\lambda_{j}\right) f_{j}\right|^{2}\right)^{\frac{1}{2}}\right\|_{p} \leq C\left\|\left(\sum_{j=1}^{\infty}\left|f_{j}\right|^{2}\right)^{\frac{1}{2}}\right\|_{p}
$$

for all possible choices of $\lambda_{j} \in \mathbb{R}^{*}$ and $f_{j} \in L^{p}\left(\mathbb{R}^{n}\right)$. Thus we only need to verify this vector-valued inequality for the two families in the theorem.

\section{Riesz transforms for the Grushin operator}

2.1. On the boundedness of $R_{j}$ and $R_{j}^{*}$. In this subsection we show that the Riesz transforms $R_{j}$ and $R_{j}^{*}$ defined in the introduction are all bounded on $L^{p}\left(\mathbb{R}^{n+1}\right)$ as long as $1<p<\infty$. This is done by showing that the operator valued functions $m_{j}(\lambda)=R_{j}(\lambda)$ and $m_{j}^{*}(\lambda)=R_{j}^{*}(\lambda)$ satisfy the conditions stated in the theorem of Weis. In view of the theorem of Weis and the equivalent condition for $\mathrm{R}$ boundedness Theorem 1.1 will follow once we prove 
Theorem 2.1. Let $T(\lambda)$ be any of the families $R_{j}(\lambda), R_{j}^{*}(\lambda), \lambda \frac{d}{d \lambda} R_{j}(\lambda)$ or $\lambda \frac{d}{d \lambda} R_{j}^{*}(\lambda)$. Then the vector valued inequality is satisfied:

$$
\left\|\left(\sum_{k=1}^{\infty}\left|T\left(\lambda_{k}\right) f_{k}\right|^{2}\right)^{\frac{1}{2}}\right\|_{p} \leq C\left\|\left(\sum_{k=1}^{\infty}\left|f_{k}\right|^{2}\right)^{\frac{1}{2}}\right\|_{p}
$$

for any $1<p<\infty$.

We only treat the cases of $R_{j}(\lambda)$ and its derivative as the other families are similarly dealt with. Without loss of generality let us assume $\lambda>0$. Recall that $R_{j}(\lambda)=A_{j}(\lambda) H(\lambda)^{-\frac{1}{2}}$ where $H(\lambda)^{-\frac{1}{2}}$ can be written in terms of the Hermite semigroup $e^{-t H(\lambda)}$ as

$$
H(\lambda)^{-\frac{1}{2}}=\int_{0}^{\infty} t^{-\frac{1}{2}} e^{-t H(\lambda)} d t
$$

An orthonormal basis for $L^{2}\left(\mathbb{R}^{n}\right)$ consisting of eigenfunctions of $H(\lambda)$ are provided by $\Phi_{\alpha}^{\lambda}(x)=\lambda^{n / 4} \Phi\left(\lambda^{\frac{1}{2}} x\right)$ where $\Phi_{\alpha}$ are the Hermite functions on $\mathbb{R}^{n}$ satisfying $H \Phi_{\alpha}=(2|\alpha|+n) \Phi_{\alpha}$. Here $H=H(1)$ is the Hermite operator. Thus $\Phi_{\alpha}^{\lambda}$ are eigenfunctions of $H(\lambda)$ with eigenvalues $(2|\alpha|+n) \lambda$. The spectral projections $P_{k}(\lambda)$ of $H(\lambda)$ are defined by

$$
P_{k}(\lambda) f=\sum_{|\alpha|=k}\left(f, \Phi_{\alpha}^{\lambda}\right) \Phi_{\alpha}^{\lambda}
$$

The kernel $h_{t}^{\lambda}(x, y)$ of the semigroup $e^{-t H(\lambda)}$ is explicitly known, thanks to Mehler's formula, and hence the kernel of $R_{j}(\lambda)$ is given by $A_{j}(\lambda) h_{t}^{\lambda}(x, y)$. It turns out that $R_{j}(\lambda)$ are all Calderon-Zygmund singular integrals. Indeed, Stempak and Torrea [7] have shown that

$$
\left|A_{j}(1) h_{t}^{1}(x, y)\right| \leq C|x-y|^{-n}
$$

and

$$
\left|\frac{\partial}{\partial x_{i}} A_{j}(1) h_{t}^{1}(x, y)\right|+\left|\frac{\partial}{\partial y_{i}} A_{j}(1) h_{t}^{1}(x, y)\right| \leq C_{i}|x-y|^{-n-1}
$$

(see Theorem 3.3 in [7]). As can be easily verified $A_{j}(\lambda) h_{t}^{\lambda}(x, y)=$ $\lambda^{\frac{n}{2}}\left(A_{j}(1) h_{t}^{1}\right)\left(\lambda^{1 / 2} x, \lambda^{1 / 2} y\right)$ and hence the kernels of $R_{j}(\lambda)$ are CalderonZygmund kernels and CZ constants are independent of $\lambda$. Now we can appeal to the vector valued inequalities for $\mathrm{CZ}$ singular integrals proved by Cordoba and Fefferman (see Theorem 1.3 Chapter XII in [1] ). Thus the Riesz transforms $R_{j}(\lambda)$ are R-bounded.

We now turn our attention to the derivative of $R_{j}(\lambda)$. Denoting the kernel of $R_{j}(1)$ by $R_{j}(x, y)$ and noting that the kernel of $R_{j}(\lambda)$ is $\lambda^{n / 2} R_{j}\left(\lambda^{1 / 2} x, \lambda^{1 / 2} y\right)$ we can easily prove the following. 
Lemma 2.2. $\lambda \frac{d}{d \lambda} R_{j}(\lambda)$ is a linear combination of $R_{j}(\lambda)$ and an operator $T_{j}(\lambda)$ given by

$$
T_{j}(\lambda) f(x)=\lambda^{n / 2} \int_{\mathbb{R}^{n}}\left(\left(x \cdot \nabla_{x}+y \cdot \nabla_{y}\right) R_{j}\right)\left(\lambda^{1 / 2} x, \lambda^{1 / 2} y\right) f(y) d y
$$

where $x \cdot \nabla_{x}=\sum_{i=1}^{n} x_{i} \frac{\partial}{\partial x_{i}}$ and $y \cdot \nabla_{y}=\sum_{i=1}^{n} y_{i} \frac{\partial}{\partial y_{i}}$.

Let $\delta_{\lambda}$ stand for the dilation operator $f(x) \rightarrow f\left(\lambda^{1 / 2} x\right)$. Then we have $T_{j}(\lambda)=\delta_{\lambda} T_{j}(1) \delta_{\lambda}^{-1}$. Integrating by parts in

$$
T_{j}(1) f(x)=\int_{\mathbb{R}^{n}}\left(\sum_{i=1}^{n}\left(x_{i} \frac{\partial}{\partial x_{i}}+y_{i} \frac{\partial}{\partial y_{i}}\right) R_{j}\right)(x, y) f(y) d y
$$

we see that it is a sum of $-R_{j}(1)$ and the commutator of $x \cdot \nabla_{x}$ with $R_{j}(1)$. Since $2 x_{i}=A_{i}(1)+A_{i}^{*}(1)$ and $2 \frac{\partial}{\partial x_{i}}=A_{i}^{*}(1)-A_{i}(1)$ we see that

$$
x \cdot \nabla_{x}=\frac{1}{4} \sum_{i=1}^{n}\left(A_{i}^{* 2}-A_{i}^{2}+\left[A_{i}, A_{i}^{*}\right]\right)=n / 2 I+\frac{1}{4} \sum_{i=1}^{n}\left(A_{i}^{* 2}-A_{i}^{2}\right)
$$

as $\left[A_{i}, A_{i}^{*}\right]=2 I$. Thus the commutator of $x \cdot \nabla_{x}$ with $R_{j}(1)$ reduces to a sum of commutators of the form $\left[A_{i}^{* 2}, R_{j}(1)\right]$ and $\left[A_{i}^{2}, R_{j}(1)\right]$ and we are left with proving

Proposition 2.3. The families $\delta_{\lambda}\left[A_{i}^{* 2}, R_{j}(1)\right] \delta_{\lambda}^{-1}$ and $\delta_{\lambda}\left[A_{i}^{2}, R_{j}(1)\right] \delta_{\lambda}^{-1}$ are $R$-bounded.

Proof. We only consider the family $\delta_{\lambda}\left[A_{i}^{2}, R_{j}(1)\right] \delta_{\lambda}^{-1}$ as the treatment of the other one is similar. We will also assume $i=j$ as the other cases are simpler as $A_{i}$ commutes with $A_{j}$ when $i$ is different from $j$. A moment's thought reveals that

$$
\left[A_{j}^{2}, R_{j}(1)\right]=A_{j}\left[A_{j}, R_{j}(1)\right]+\left[A_{j}, R_{j}(1)\right] A_{j}
$$

and hence we are left with the families

$$
\delta_{\lambda} A_{j}\left[A_{j}, R_{j}(1)\right] \delta_{\lambda}^{-1}, \delta_{\lambda}\left[A_{j}, R_{j}(1)\right] A_{j} \delta_{\lambda}^{-1}
$$

and again we will consider only the first family.

As

$$
A_{j}\left[A_{j}, R_{j}(1)\right]=A_{j}^{3} H^{-1 / 2}-A_{j}^{2} H^{-1 / 2} A_{j}=A_{j}^{2}\left[A_{j}, H^{-1 / 2}\right]
$$

making use of the fact that $H^{-1 / 2} A_{j}=A_{j}(H+2)^{-1 / 2}$ we get

$$
A_{j}\left[A_{j}, R_{j}(1)\right]=-A_{j}^{3}\left((H+2)^{-1 / 2}-H^{-1 / 2}\right) .
$$

An easy calculation shows that

$$
\delta_{\lambda} A_{j} \delta_{\lambda}^{-1}=\lambda^{-1 / 2} A_{j}(\lambda), \delta_{\lambda} H^{-1 / 2} \delta_{\lambda}^{-1}=\lambda^{1 / 2} H(\lambda)^{-1 / 2}
$$


and hence we finally get

$$
\delta_{\lambda} A_{j}\left[A_{j}, R_{j}(1)\right] \delta_{\lambda}^{-1}=-\lambda^{-1} A_{j}(\lambda)^{3}\left((H(\lambda)+2 \lambda)^{-1 / 2}-H(\lambda)^{-1 / 2}\right) .
$$

The above can be rewritten as

$$
A_{j}(\lambda)^{3} \int_{0}^{1}(H(\lambda)+2 \lambda s)^{-3 / 2} d s .
$$

Thus everything boils down to showing that the family $A_{j}(\lambda)^{3}(H(\lambda)+$ $2 \lambda s)^{-3 / 2}$ is R-bounded uniformly in $s, 0<s<1$. But these operators are also $\mathrm{CZ}$ singular integrals whose kernels satisfy estimates uniformly in $\lambda$ and $s$. This completes the proof.

2.2. Higher order Riesz transforms. We briefly recall some notations we use referring to $\left[\mathbf{9}\right.$ for details. Given a function $f$ on $\mathbb{C}^{n}$ we denote by $W_{\lambda}(f)$ the Weyl transform defined by

$$
W_{\lambda}(f)=\int_{\mathbb{C}^{n}} f(z) \pi_{\lambda}(z, 0) d z
$$

where $\pi_{\lambda}$ stands for the Schrödinger representation of the Heisenberg group realised on $L^{2}\left(\mathbb{R}^{n}\right)$. For $P \in \mathcal{H}_{p, q}$ the operator $G_{\lambda}(P)=W_{\lambda}\left(\mathcal{F}_{\lambda} P\right)$ is called its Weyl correspondence. Here $\mathcal{F}_{\lambda}$ stands for the symplectic Fourier transform. The unitary group $U(n)$ acts on $\mathcal{H}_{p, q}$ by $\rho(\sigma) P(z)=$ $P\left(\sigma^{-1} z\right)$ and the action is irreducible, i.e. we get an irreducible unitary representation of $U(n)$ on $\mathcal{H}_{p, q}$. In view of this, if $P \in \mathcal{H}_{p, q}$ then the linear span of its orbit $\rho(\sigma) P$ under $U(n)$ is the whole of $\mathcal{H}_{p, q}$. Therefore, if we let $P_{0}(z)=z_{1}^{p}{\overline{z_{2}}}^{q}$ then any $P \in \mathcal{H}_{p, q}$ is a linear combination of $\rho(\sigma) P_{0}, \sigma \in U(n)$.

Proposition 2.4. If the higher order Riesz transform $R_{P_{0}}$ is bounded on $L^{p}\left(\mathbb{R}^{n+1}\right)$ then so is $R_{P}$ for any $P \in \mathcal{H}_{p, q}$.

PROOF. In order to prove this proposition we need to use several facts about the symplectic group $S p(n, \mathbb{R})$ and the metaplectic representations $\mu_{\lambda}(\sigma)$ of $S p(n, \mathbb{R})$. A good source for the material we use here is Chapter 4 of Folland [3]. The action of $U(n)$ on the Heisenberg group leads to certain unitary operators, denoted by $\mu_{\lambda}(\sigma)$ so that

$$
\mu_{\lambda}(\sigma) \pi_{\lambda}(z, t) \mu_{\lambda}(\sigma)^{*}=\pi_{\lambda}(\sigma z, t) .
$$

Recalling the definition of $R_{P}$ we see that this means

$$
R_{\rho(\sigma) P} f(x, t)=\frac{1}{2 \pi} \int_{-\infty}^{\infty} e^{-i \lambda t} \mu_{\lambda}(\sigma) G_{\lambda}(P) \mu_{\lambda}(\sigma)^{*} f^{\lambda}(x) d \lambda .
$$

Therefore, our proposition will be proved if we show that the operators

$$
f \rightarrow \frac{1}{2 \pi} \int_{-\infty}^{\infty} e^{-i \lambda t} \mu_{\lambda}(\sigma) f^{\lambda}(x) d \lambda
$$


are all bounded on $L^{p}\left(\mathbb{R}^{n+1}\right)$.

If we identify $\mathbb{C}^{n}$ with $\mathbb{R}^{2 n}$ the group $U(n)$ corresponds to $S p(n, \mathbb{R}) \cap$ $O(2 n, \mathbb{R})$. By Proposition 4.10 in 3 the group $S p(n, \mathbb{R})$ is generated by certain subgroups $N=\left\{\left(\begin{array}{cc}I & 0 \\ C & I\end{array}\right): C=C^{*}\right\}$ and $D=\left\{\left(\begin{array}{cc}A & 0 \\ 0 & A^{*-1}\end{array}\right): A \in G L(n, \mathbb{R})\right\}$ and the matrix $J=\left(\begin{array}{cc}0 & I \\ -I & 0\end{array}\right)$. For elements of these subgroups $\mu_{\lambda}(\sigma)$ are explicitly known. In fact we have:

$$
\mu_{\lambda}(\sigma) \varphi(\xi)=|A|^{-1 / 2} \varphi\left(A^{-1} \xi\right)
$$

if $\sigma \in D$ and

$$
\mu_{\lambda}(\sigma) \varphi(\xi)=e^{\frac{i}{2} \lambda \xi \cdot C \xi} \varphi(\xi)
$$

if $\sigma \in N$. Moreover,

$$
\mu_{\lambda}(J) \varphi(\xi)=\hat{\varphi}(\lambda \xi)
$$

is the Fourier transform on $\mathbb{R}^{n}$ followed by a dilation. Clearly, the operator

$$
f \rightarrow \frac{1}{2 \pi} \int_{-\infty}^{\infty} e^{-i \lambda t}|A|^{-1 / 2} f^{\lambda}\left(A^{-1} x\right) d \lambda=|A|^{-1 / 2} f\left(A^{-1} x, t\right)
$$

is bounded on $L^{p}\left(\mathbb{R}^{n+1}\right)$. And so is the operator

$$
f \rightarrow \frac{1}{2 \pi} \int_{-\infty}^{\infty} e^{-i \lambda t} e^{\frac{i}{2} \lambda \xi \cdot C \xi} f^{\lambda}(x) d \lambda=f\left(x, t-\frac{1}{2} x \cdot C x\right) .
$$

Though Fourier transform does not define a bounded operator on $L^{p}$ unless $p=2$ we can take care of the operators involving $\mu_{\lambda}(J)$ in the following way. Observe that we need to prove the boundedness of

$$
f \rightarrow \frac{1}{2 \pi} \int_{-\infty}^{\infty} e^{-i \lambda t} \mu_{\lambda}(J) G_{\lambda}(P) \mu_{\lambda}(J)^{*} f^{\lambda}(x) d \lambda
$$

whenever the operator

$$
f \rightarrow \frac{1}{2 \pi} \int_{-\infty}^{\infty} e^{-i \lambda t} G_{\lambda}(P) f^{\lambda}(x) d \lambda
$$

is bounded. But as $P \in \mathcal{H}_{p, q}$ is homogeneous of bidegree $(p, q)$ and the action of $J$ is $\rho(J) P(z)=P(-i z)=(-i)^{p-q} P(z)$ the former operator is just a scalar multiple of the latter and hence bounded. As $S p(n, \mathbb{R})$ is generated by $N, D$ and $J$ the proposition is proved.

In view of the above proposition Theorem 1.2 will be proved once we obtain the following result.

Theorem 2.5. Let $P_{0}(z)=z_{1}^{p} \overline{z_{2}}{ }^{q}$. Then $R_{P_{0}}$ is bounded on $L^{p}\left(\mathbb{R}^{n+1}\right)$ for $1<p<\infty$. 
Proof. When $P_{0}(z)=z_{1}^{p} \bar{z}_{2}^{q}, G_{\lambda}\left(P_{0}\right)$ given by $A_{2}(\lambda)^{q} A_{1}(\lambda)^{* p}$, see Geller [4] or [9]. Hence the multiplier corresponding to $R_{P_{0}}$ is given by $A_{2}(\lambda)^{q} A_{1}(\lambda)^{* p} H(\lambda)^{-\frac{p+q}{2}}$. We prove the boundedness of $R_{P_{0}}$ by showing that this multiplier can be written as the product of the multipliers corresponding to first order Riesz transform and their adjoints. Since $A_{2}(\lambda) A_{1}(\lambda)^{*}$ commutes with $H(\lambda)$, we can write $A_{2}(\lambda)^{q} A_{1}(\lambda)^{* p} H(\lambda)^{-\frac{p+q}{2}}$ as the product of operators of the form $H(\lambda)^{-1 / 2} A_{2}(\lambda), A_{1}(\lambda)^{*} H(\lambda)^{-1 / 2}$ and $\lambda H(\lambda)^{-1}$. We refer to [6] for details. $\lambda H(\lambda)^{-1}$ can be written as

$$
\frac{1}{2} H(\lambda)^{-\frac{1}{2}}\left[A_{1}^{*}(\lambda) A_{1}(\lambda)-A_{1}(\lambda) A_{2}(\lambda)^{*}\right] H(\lambda)^{-\frac{1}{2}} .
$$

$A_{1}(\lambda)^{*} H(\lambda)^{-1 / 2}$ is the multiplier corresponding to the first order Riesz transform $R_{1}^{*}(\lambda)$ and $H(\lambda)^{-1 / 2} A_{2}(\lambda)$ is the multiplier corresponding to the adjoint of $R_{2}^{*}(\lambda)$. The proof is now complete as we have already shown that the operators corresponding to multipliers of these forms are bounded.

\section{On the boundedness of multiplier transforms}

In this section we prove Theorem 1.3. In view of Theorem 1.5 it is enough to show that the families $m(H(\lambda))$ and $\lambda \frac{d}{d \lambda} m(H(\lambda))$ are R-bounded. We remark that we only need to prove vector valued inequalities for these families.

3.1. R-boundedness of the family $m(H(\lambda))$. For a fixed $\lambda$ conditions on $m$ are known so that $m(H(\lambda))$ is bounded on $L^{p}\left(\mathbb{R}^{n}\right), 1<$ $p<\infty$, see e.g. Theorem 4.2.1 in [9]. Under the same assumptions on $m$ it is possible to prove a vector valued inequality for the sequence of operators $m\left(H\left(\lambda_{j}\right)\right)$ for any choice of $\lambda_{j}$.

Theorem 3.1. Assume that $m \in C^{k}\left(\mathbb{R}^{*}\right)$ satisfies $\left|m^{(j)}(\lambda)\right| \leq C_{j}|\lambda|^{-j}$ for large $|\lambda|$ and for all $j=0,1,2, . ., k$ where $k \geq n / 2$. Then for any choice of $\lambda_{j} \in \mathbb{R}^{*}$ and $f_{j} \in L^{p}\left(\mathbb{R}^{n}\right), 1<p<\infty$ we have the uniform estimate

$$
\left\|\left(\sum_{j=0}^{\infty}\left|m\left(H\left(\lambda_{j}\right)\right) f_{j}\right|^{2}\right)^{1 / 2}\right\|_{p} \leq C\left\|\left(\sum_{j=0}^{\infty}\left|f_{j}\right|^{2}\right)^{1 / 2}\right\|_{p} .
$$

In proving this theorem we closely follow the proof of Theorem 4.2.1 in [9] and hence we briefly recall the proof. Fixing $\lambda>0$ for the sake of definiteness we consider the boundedness of $m(H(\lambda))$ on $L^{p}\left(\mathbb{R}^{n}\right)$. In $[\mathbf{9}$ this is achieved by means of Littlewood-Paley g-functions. We define, 
for each positive integer $k$, the function

$$
\left(g_{k}^{\lambda}(f, x)\right)^{2}=\int_{0}^{\infty}\left|\partial_{t}^{k} T_{t}^{\lambda} f(x)\right|^{2} t^{2 k-1} d t
$$

where $T_{t}^{\lambda}=e^{-t H(\lambda)}$ is the semigroup generated by $H(\lambda)$. In [9] it is shown that (see Theorem 4.1.2) $g_{1}^{\lambda}(f)$ can be considered as a singular integral operator whose kernel is taking values in the Hilbert space $L^{2}\left(\mathbb{R}^{+}, t d t\right)$ and hence bounded on $L^{p}\left(\mathbb{R}^{n}\right)$. Moreover, it is also shown that $g_{k}^{\lambda}(f)$ is a constant multiple of isometry on $L^{2}\left(\mathbb{R}^{n}\right)$ and hence we have the equivalence of norms:

$$
C_{1}\|f\|_{p} \leq\left\|g_{1}^{\lambda}(f)\right\|_{p} \leq C_{2}\|f\|_{p}
$$

for all $1<p<\infty$. Here, it can be verified that the constants $C_{j}, j=1,2$ can be taken independent of $\lambda$. Moreover, we also have the pointiest estimate $g_{k}^{\lambda}(f, x) \leq C_{k} g_{k+1}^{\lambda}(f, x)$.

A version of Cordoba-Fefferman theorem for CZ singular integrals whose kernels are taking values in a Hilbert space is true. In fact an easy modification of the proof given in Torchinsky [11] substantiates this claim. By treating the g-functions as singular integral operators with kernels taking values in $L^{2}\left(\mathbb{R}^{+}, t d t\right)$ we can deduce the following result.

Theorem 3.2. For any choice of $\lambda_{j} \in \mathbb{R}^{*}$ and $f_{j} \in L^{p}\left(\mathbb{R}^{n}\right)$ we have

$$
\left\|\left(\sum_{j=0}^{\infty}\left|g_{1}^{\lambda_{j}}\left(f_{j}\right)\right|^{2}\right)^{1 / 2}\right\|_{p} \leq C\left\|\left(\sum_{j=0}^{\infty}\left|f_{j}\right|^{2}\right)^{1 / 2}\right\|_{p}
$$

for all $1<p<\infty$. The reverse inequality also holds.

In order to prove the multiplier theorem we also need to consider $g_{k}^{* \lambda}$ functions which are defined by

$$
\left(g_{k}^{* \lambda}(f, x)\right)^{2}=\int_{\mathbb{R}^{n}} \int_{0}^{\infty} t^{-n / 2}\left(1+t^{-1}|x-y|^{2}\right)^{-k}\left|\partial_{t} T_{t}^{\lambda} f(y)\right|^{2} t d t d y .
$$

If $k>n / 2$ it is proved in [9] (see Theorem 4.1.3) that

$$
\left\|g_{k}^{* \lambda}(f)\right\|_{p} \leq C\|f\|_{p}, p>2 .
$$

The proof of this depends on two facts: the boundedness of $g_{1}^{\lambda}$ functions and the Hardy-Littlewood maximal functions. By the celebrated theorem of Fefferman-Stein the Hardy-Littlewood maximal function satisfies a vector valued inequality, see Theorem 1.1, Chapter XII in [11. Hence by combining this with Theorem 3.2 above we get 
Theorem 3.3. For any choice of $\lambda_{j} \in \mathbb{R}^{*}$ and $f_{j} \in L^{p}\left(\mathbb{R}^{n}\right)$ we have

$$
\left\|\left(\sum_{j=0}^{\infty}\left|g_{k}^{* \lambda_{j}}\left(f_{j}\right)\right|^{2}\right)^{1 / 2}\right\|_{p} \leq C\left\|\left(\sum_{j=0}^{\infty}\left|f_{j}\right|^{2}\right)^{1 / 2}\right\|_{p}
$$

for all $p>2$ provided $k>n / 2$.

Under the hypothesis on $m$ it has been proved in [9] (see Section 4.2) that the pointwise inequality

$$
g_{k+1}^{\lambda}(m(H(\lambda)) f, x) \leq C_{k} g_{k}^{* \lambda}(f, x)
$$

holds. It can be checked, by following the proof carefully, that the constant $C_{k}$ can be taken independent of $\lambda$. It is then clear that a vector valued analogue of the above estimate is valid. Hence, by appealing to Theorems 3.2 and 3.3 we obtain Theorem 3.1. This takes care of the R-boundedness of $m(H(\lambda))$.

3.2. R-boundedness of the derivative of $m(H(\lambda))$. We begin with the observation that the kernel of $m(H(\lambda))$ is given by

$$
\lambda^{n / 2} \sum_{k=0}^{\infty} m((2 k+n) \lambda) \Phi_{k}\left(\lambda^{1 / 2} x, \lambda^{1 / 2} y\right)
$$

where $\Phi_{k}(x, y)$ is the kernel of $P_{k}$. If we let $m_{\lambda}(t)=m(t \lambda)$ then, with obvious notations, we have

$$
m(H(\lambda))(x, y)=\lambda^{n / 2} m_{\lambda}(H)\left(\lambda^{1 / 2} x, \lambda^{1 / 2} y\right) .
$$

Using this we can easily prove

Lemma 3.4. $\lambda \frac{d}{d \lambda} m(H(\lambda))$ is a linear combination of operators of the form $m(H(\lambda)), H(\lambda) m^{\prime}(H(\lambda))$ and the commutators $\delta_{\lambda}\left[A_{j}^{2}, m_{\lambda}(H)\right] \delta_{\lambda}^{-1}$ and $\delta_{\lambda}\left[A_{j}^{* 2}, m_{\lambda}(H)\right] \delta_{\lambda}^{-1}$.

We have just shown that the families $m(H(\lambda))$ and $H(\lambda) m^{\prime}(H(\lambda))$ are R-bounded under the hypothesis of Theorem 1.3. To handle the commutators we proceed as in the case of Riesz transforms. We need to consider the operators $\delta_{\lambda} A_{j}\left[A_{j}, m_{\lambda}(H)\right] \delta_{\lambda}^{-1}$ and $\delta_{\lambda}\left[A_{j}, m_{\lambda}(H)\right] A_{j} \delta_{\lambda}^{-1}$. As before using $m_{\lambda}(H) A_{j}=A_{j} m_{\lambda}(H+2)$ we get

$$
\delta_{\lambda} A_{j}\left[A_{j}, m_{\lambda}(H)\right] \delta_{\lambda}^{-1}=-\delta_{\lambda} A_{j}^{2}\left(m_{\lambda}(H+2)-m_{\lambda}(H)\right) \delta_{\lambda}^{-1} .
$$

Finally, the above can be written in the form

$$
\left.A_{j}(\lambda)^{2} \int_{0}^{1} m^{\prime}(H(\lambda)+2 \lambda s)\right) d s .
$$


Since $A_{j}(\lambda)^{2} H(\lambda)^{-1}$ are singular integral operators we need to check the R-boundedness of

$$
\left.\int_{0}^{1} H(\lambda) m^{\prime}(H(\lambda)+2 \lambda s)\right) d s
$$

The hypothesis on $m$ allows us to handle this family with estimates uniformly in $s, 0<s<1$. This completes the proof of the R-boundedness of $\lambda \frac{d}{d \lambda} M(H(\lambda))$ and hence Theorem 1.3 is proved.

3.3. Boundedness of the Bochner-Riesz means. Recall that the kernel of $P_{k}(\lambda)$ is of the form $|\lambda|^{n / 2} \Phi_{k}\left(|\lambda|^{1 / 2} x,|\lambda|^{1 / 2} y\right)$ and hence

$$
B_{R}^{\delta} f(x, t)=\frac{1}{2 \pi} \int_{-\infty}^{\infty} \int_{\mathbb{R}^{n}} e^{-i \lambda t}|\lambda|^{n / 2} s_{R /|\lambda|}^{\delta}\left(|\lambda|^{1 / 2} x,|\lambda|^{1 / 2} y\right) f^{\lambda}(y) d y d \lambda .
$$

where

$$
s_{R}^{\delta}(x, y)=\sum_{(2 k+n) \leq R}(1-(2 k+n) / R)_{+}^{\delta} \Phi_{k}(x, y) .
$$

If $D_{r} f(x, t)=r^{n+2} f\left(r x, r^{2} t\right)$ stands for the nonisotropic dilation, then it is easy to see that

$$
B_{R}^{\delta} D_{r} f(x, t)=D_{r} B_{R / r^{2}}^{\delta} f(x, t)
$$

and hence it is enough to prove the boundedness of $B_{1}^{\delta}$. Note that

$$
B_{1}^{\delta} f(x, t)=\frac{1}{2 \pi} \int_{-\infty}^{\infty} e^{-i \lambda t} m_{\delta}(\lambda) f^{\lambda}(x) d \lambda
$$

where

$$
m_{\delta}(\lambda)=\sum_{(2 k+n)|\lambda| \leq 1}(1-(2 k+n)|\lambda|)^{\delta} P_{k}(\lambda) .
$$

Thus we can view $B_{1}^{\delta}$ as a Fourier multiplier corresponding to the operator valued multiplier $m_{\delta}(\lambda)$. Therefore, in order to prove Theorem 1.4 we only need to show that $m_{\delta}(\lambda)$ and $\lambda \frac{d}{d \lambda} m_{\delta}(\lambda)$ are both R-bounded families of operators.

Let

$$
S_{R}^{\delta} f(x)=\sum_{(2 k+n) \leq R}(1-(2 k+n) / R)_{+}^{\delta} P_{k} f
$$

stand for the Bochner-Riesz means associated to the Hermite operator $H$. Then it follows that

$$
m_{\delta}(\lambda)=\delta_{|\lambda|} S_{|\lambda|-1}^{\delta} \delta_{|\lambda|}^{-1} .
$$

We make use of the following result in order to prove the R-boundedness of the family $m_{\delta}(\lambda)$. Let $M f$ stand for the Hardy-Littlewood maximal function of $f$. 
Theorem 3.5. Assume that $f \in L^{p}\left(\mathbb{R}^{n}\right), 1 \leq p \leq \infty$ and $\delta>(n-$ 1) $/ 2+1 / 6$. Then $\sup _{R>0}\left|S_{R}^{\delta} f(x)\right| \leq C(M f(x)+M f(-x))$.

This theorem can be proved using the estimates given in [9] (see Theorem 3.3.5). Details can be found in [8]. Using this result it is not difficult to prove

Theorem 3.6. The family $m_{\delta}(\lambda)$ is $R$-bounded on $L^{p}\left(\mathbb{R}^{n}\right)$ for all $\delta>$ $(n-1) / 2+1 / 6$ and $1<p<\infty$.

PROOF. In view of the relation between $m_{\delta}(\lambda)$ and $S_{R}^{\delta}$ we need to show that

$$
\left\|\left(\sum_{j=1}^{\infty}\left|\delta_{R_{j}}^{-1} S_{R_{j}}^{\delta} \delta_{R_{j}} f_{j}\right|^{2}\right)^{1 / 2}\right\|_{p} \leq C\left\|\left(\sum_{j=1}^{\infty}\left|f_{j}\right|^{2}\right)^{1 / 2}\right\|_{p}
$$

for all sequences $R_{j}>0$ and $f_{j} \in L^{p}\left(\mathbb{R}^{n}\right)$. By the result of Theorem 3.5

$$
\left|\delta_{R_{j}}^{-1} S_{R_{j}}^{\delta} \delta_{R_{j}} f_{j}(x)\right| \leq C\left(M\left(\delta_{R_{j}} f_{j}\right)\left(R_{j}^{-1 / 2} x\right)+M\left(\delta_{R_{j}} f_{j}\right)\left(-R_{j}^{-1 / 2} x\right)\right)
$$

and a simple calculation, recalling the definition of $M f$, shows that

$$
\left|\delta_{R_{j}}^{-1} S_{R_{j}}^{\delta} \delta_{R_{j}} f_{j}(x)\right| \leq C M f_{j}(x) .
$$

Thus we are required to prove

$$
\left\|\left(\sum_{j=1}^{\infty}\left|M f_{j}\right|^{2}\right)^{1 / 2}\right\|_{p} \leq C\left\|\left(\sum_{j=1}^{\infty}\left|f_{j}\right|^{2}\right)^{1 / 2}\right\|_{p}
$$

which is the Fefferman-Stein inequality for the maximal function.

It remains to be proved that the family $\lambda \frac{d}{d \lambda} m_{\delta}(\lambda)$ is also R-bounded. This is done in the following theorem.

Theorem 3.7. For $\delta>(n+1) / 2+1 / 6$ the family $\lambda \frac{d}{d \lambda} m_{\delta}(\lambda)$ is $R$ bounded on $L^{p}\left(\mathbb{R}^{n+1}\right), 1<p<\infty$.

Proof. Applying Lemma 3.4 with $m(t)=(1-t)_{+}^{\delta}$ we see that $\lambda \frac{d}{d \lambda} m_{\delta}(\lambda)$ is a linear combination of $(1-H(\lambda))_{+}^{\delta}, H(\lambda)\left((1-H(\lambda))_{+}^{\delta-1}\right.$ and commutators of the form $\left[A_{j}(\lambda)^{2},(1-H(\lambda))_{+}^{\delta}\right]$ and $\left[A_{j}(\lambda)^{* 2},(1-\right.$ $\left.H(\lambda))_{+}^{\delta}\right]$. The first two families are handled as in the previous theorem. We prove the R-boundedness of $\left[A_{j}(\lambda)^{2},(1-H(\lambda))_{+}^{\delta}\right]$ and the other family can be similarly handled. It is enough to look at $A_{j}(\lambda)^{2}(1-$ $H(\lambda))_{+}^{\delta}$ which we write as

$$
A_{j}(\lambda)^{2}(1-H(\lambda))_{+}^{\delta}=A_{j}(\lambda)^{2} H(\lambda)^{-1} H(\lambda)(1-H(\lambda))_{+}^{\delta} .
$$


But $A_{j}(\lambda)^{2} H(\lambda)^{-1}$ are singular integral operators and

$$
H(\lambda)(1-H(\lambda))_{+}^{\delta}=(1-H(\lambda))_{+}^{\delta}-(1-H(\lambda))_{+}^{\delta+1} .
$$

Thus $A_{j}(\lambda)^{2}(1-H(\lambda))_{+}^{\delta}$ is R-bounded.

\section{Acknowledgments}

The work of the last author is supported by J. C. Bose Fellowship from the Department of Science and Technology (DST).

\section{References}

[1] T. Coulhon, D . Mueller and J. Zienkiewicz, About Riesz transforms on Heisenberg groups, Math. Annalen 312(1996), 369-379.

[2] A. F. M. ter Elst, D. W. Robinson and A. Sikora, Heat kernels and Riesz transforms on nilpotent Lie groups, Colloq. Math. 74 (1997), 191-218.

[3] G. B. Folland, Harmonic analysis in phase space, Annals of Mathematics Studies, 122. Princeton University Press, Princeton, NJ, 1989.

[4] D. Geller, Spherical harmonics, the Weyl transform and the Fourier transform on the Heisenberg group, Can. J. Math. 36(1984), 615-684.

[5] R. Meyer, $L^{p}$ estimates for the Grushin operator, arXiv:0709.2188 (2007)

[6] P. K. Sanjay and S. Thangavelu, Revisiting Riesz transforms on Heisenberg groups, preprint.

[7] K. Stempak and J. L. Torrea, Poisson integrals and Riesz transforms for the Hermite function expansions with weights, J. Funct. Anal. 202 (2003), 443-472.

[8] S. Thangavelu, Summability of Hermite expansions I,II, Trans. Amer. Math. Soc. 314 (1989), 119-170.

[9] S. Thangavelu, Lectures on Hermite and Laguerre expansions, Math. Notes No. 42, Princeton Univ. Press (1993).

[10] S. Thangavelu, An introduction to the uncertainty principle, Prog. Math. 217(2004), Birkhauser-Boston.

[11] A. Torchinsky, Real variable methods in harmonic analysis, Dover publications, Inc., Mineola, New York (2004)

[12] L. Weis, Operator valued Fourier multiplier theorems and maximal $L^{p}$ regularity, Math. Ann. 319 (2001), 735-758.

Department of Mathematics, Indian Institute of Science, Bangalore560012

E-mail address: jyoti@math.iisc.ernet.in, sanjay@math.iisc.ernet.in, veluma@math.iisc.ernet.in 